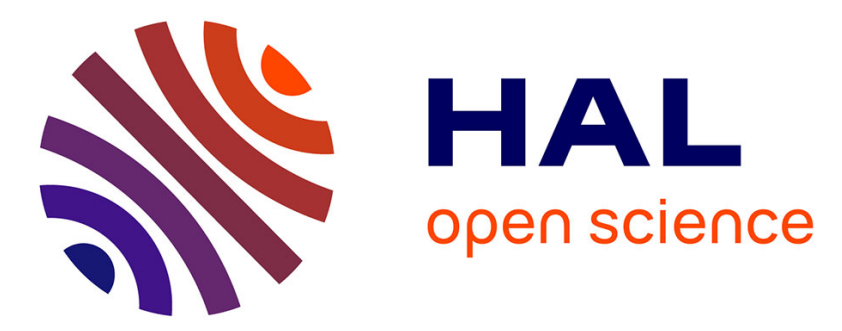

\title{
Mise au point et étude d'un test enzymatique de la digestibilité de fourrages pauvres ou riches en amidon
}

\author{
Marc Lila, Yves Barrière, Rémi Traineau, Simone Allerit
}

\section{To cite this version:}

Marc Lila, Yves Barrière, Rémi Traineau, Simone Allerit. Mise au point et étude d'un test enzymatique de la digestibilité de fourrages pauvres ou riches en amidon. Agronomie, 1986, 6 (3), pp.285-291. hal00884877

\section{HAL Id: hal-00884877 \\ https://hal.science/hal-00884877}

Submitted on 1 Jan 1986

HAL is a multi-disciplinary open access archive for the deposit and dissemination of scientific research documents, whether they are published or not. The documents may come from teaching and research institutions in France or abroad, or from public or private research centers.
L'archive ouverte pluridisciplinaire HAL, est destinée au dépôt et à la diffusion de documents scientifiques de niveau recherche, publiés ou non, émanant des établissements d'enseignement et de recherche français ou étrangers, des laboratoires publics ou privés. 


\title{
Mise au point et étude d'un test enzymatique de la digestibilité de fourrages pauvres ou riches en amidon
}

\author{
Marc LILA, Yves BARRIÈRE \& Rémi TRAINEAU \\ avec la collaboration technique de Simone ALLERIT \\ I.N.R.A., Station d'Amélioration des Plantes fourragères, F 86600 Lusignan
}

RÉSUMÉ

\begin{abstract}
Un test enzymatique de digestibilité des fourrages à partir d'enzymes (amylase, pepsine, cellulase) a été mis au point ; son protocole est détaillé.

Sur maïs ensilage, ce test est assez bien corrélé avec la méthode classique qui utilise du jus de rumen et il est plus précis et plus fidèle. D'autres essais ont montré que ces résultats étaient également valables sur des fourrages pauvres en amidon comme le sorgho ou la fétuque élevée. Sur maïs, ce test permet de mettre en évidence des différences nettes de digestibilité, également mises en évidence par des mesures avec moutons, alors que la corrélation entre ces deux mesures n'est pas bonne quand les différences variétales ne sont pas significatives. Il semble directement utilisable pour la sélection des fourrages pour la qualité si l'on souhaite soit faire des gains notables de qualité, soit éliminer des génotypes à trop faible digestibilité, soit vérifier que l'on ne perd pas en qualité au cours de cycles d'amélioration du rendement ou de la précocité.
\end{abstract}

Mots clés additionnels : Maïs, fétuque, sorgho.

An in vitro enzymatic test of forage digestibility is described, using amylolytic, proteinolytic and cellulolytic enzymes. The detailed method is given in an appendix. With maize silage, this APC method gave results correlated with those of the classic rumen juice method, and was more precise and reproducible. Other trials showed that this method also gave good results with low starch-content forages such as tall fescue or sudangrass or sorghum $\mathrm{x}$ sudangrass hybrids. With maize, correlations between the APC test of digestibility and digestibility measured by sheep were not good if the variability between test varieties was not significant. The test could be used in breeding programmes if the aim is either to increase digestibility significantly, or to discard low digestibility genotypes or to test that there is no decrease in digestibility during breeding cycles for yield or earliness.

Additional key words : Maize, corn, fescue, sudangrass, sorghum x sudangrass hybrids.

\section{INTRODUCTION}

Les fourrages cultivés représentent actuellement en France environ 6000000 ha, dont le quart en maîs. La sélection des plantes fourragères comprend classiquement des aspects productivité, précocité, résistance aux maladies, éventuellement pérennité, mais la valeur alimentaire, pour les ruminants essentiellement, est aussi importante. Le tri pour la digestibilité de nombreuses descendances nécessite un test de laboratoire fiable, fidèle, représentatif, facile à mettre en neuvre et aussi peu onéreux que possible. Si la référence zootechnique pour l'appréciation de la digestibilité des fourrages reste le «mouton castré standard», animal qui n'est pas en production, la méthode la plus classi- que d'appréciation en grande série de cette digestibilité est sans doute le test réalisé in vitro avec du jus de rumen prélevé sur des bovins fistulisés. Cette technique présente des inconvénients de plusieurs natures liés à l'entretien des animaux, à l'absence de caractères «standards» du jus prélevé, à une appréciation différente de la digestibilité en fonction de la teneur en amidon du fourrage testé. Pour pallier ces défauts de la méthode jus de rumen et tenter d'apprécier une digestibilité potentielle totale du fourrage testé quelle que soit sa teneur en amidon, on a développé à Lusignan un test de prévision de la digestibilité des fourrages applicable aussi bien à des fourrages pauvres qu'à des fourrages riches en amidon, test réalisé à partir d'enzymes. 
Ce test A.P.C. consiste à faire agir successivement sur le broyat de fourrage une amylase, une pepsine et une cellulase qui réalisent, en principe, la digestion de l'ensemble des éléments nutritifs susceptibles d'être digérés par un ruminant. Des essais de cette méthode ont été réalisés sur différentes espèces (sorgho, maïs, fétuque...). Nous décrirons plus particulièrement ici une expérimentation faite sur maîs récolté au stade ensilage, soit en plante entière (fourrage « riche » en amidon), soit après élimination de l'épi (fourrage « pauvre " en amidon), en présentant également de façon plus succincte des résultats obtenus sur sorgho et fétuque (fourrages pauvres en amidon).

\section{MATÉRIEL ET MÉTHODES}

\section{A. Tests de digestibilité}

\section{Jus de rumen (digestibilité in vitro)}

Cette technique qui, depuis sa mise au point par TILLEY \& TERRY (1963) a subi de nombreuses variantes, reste basée sur le même principe (LILA, 1977). L'échantillon subit d'abord une digestion cellulolytique par les microorganismes présents dans le jus de rumen, ensuite une digestion pepsique permet de solubiliser ces microorganismes ainsi que les protéines végétales non digérées. La digestion peut se réaliser aussi bien en éprouvette qu'en sachet de nylon plongé dans le jus de rumen à condition bien entendu de respecter les conditions de $\mathrm{pH}$, de température $\left(38^{\circ}\right)$ et d'anaérobiose, mais surtout de surveiller l'alimentation et l'état de santé des animaux fistulés donnant le jus de rumen, animaux nourris exclusivement d'un bon foin de luzerne.

Dans notre expérience, nous avons utilisé la technique suivante :

- les échantillons séchés préalablement à $80^{\circ} \mathrm{C}$ maximum puis broyés au broyeur à marteau pour passer au travers d'un tamis de $0,5 \mathrm{~mm}$ sont pesés secs (500 mg) et introduits dans une éprouvette ;

- immédiatement après prélèvement du jus de rumen, celui-ci est mélangé dans la proportion de 1 pour 4 à une solution tampon ou salive artificielle de MAC DOUGALL (1948) saturée en gaz carbonique $(\mathrm{pH} \mathrm{7)}$;

- $50 \mathrm{ml}$ de mélange sont distribués dans les éprouvettes rapidement bouchées et mises à l'étuve à $38^{\circ} \mathrm{C}$. Dans le cas du maïs, fourrage à faible teneur en azote, on ajoute $10 \mathrm{ml}$ d'une solution de $\left(\mathrm{NH}_{4}\right)_{2} \mathrm{SO}_{4}$ à $4,56 \mathrm{~g} / \mathrm{l}$;

- après $48 \mathrm{~h}$ d'incubation, on ajoute de l'acide chlorhydrique qui, ramenant le $\mathrm{pH}$ à 1 , tue les microorganismes du rumen. La pepsine est alors introduite et une nouvelle incubation de $48 \mathrm{~h}$ est mise en route ;

- à la fin de cette $2^{\mathrm{e}}$ digestion, le résidu est récupéré sur creusets filtrants préalablement tarés ;

- le résidu séché à l'étuve est pesé et, par différence avec le poids de l'échantillon de fourrage mis au départ, on détermine la quantité digérée que l'on exprime en pourcentage de la matière sèche initiale.

Il est recommandé d'ajouter des «blancs " dans la série (éprouvettes sans fourrage mais recevant toutes les solutions) et de corriger éventuellement la quantité de résidu par la valeur d'un «blanc moyen » avant d'effectuer la différence.

\section{A.P.C. : amylase, pepsine, cellulase}

Cette méthode consiste à faire agir successivement ces 3 enzymes diluées dans des tampons appropriés sur les échantillons de fourrage placés dans des sachets de tissu de nylon. L'ensemble des sachets de la série est plongé dans un bain contenant les différentes solutions d'enzymes. Ceci est une adaptation de la technique décrite par AUFRÈRE (1982) ; seul un prétraitement des échantillons par une amylase précède les traitements à la pepsine puis à la cellulase.

Le mode opératoire complet de cette technique nouvellement mise au point est détaillé ci-dessous.

\section{a) Matériel nécessaire à la réalisation du test}

- Etuve réglable de 39 à $60{ }^{\circ} \mathrm{C}$.

- Balance au $1 \mathrm{mg}$.

- Tissu de nylon, maille $20 \mu$ réf. : $5025 \mathrm{M}$ Beck et Michel (5, allée des Bourbonnais, 78310 Maurepas).

- Soudeuse à plastique pour la confection des sachets.

- Bécher de 21 pour incubation.

\section{b) Réactifs employés}

(1) Amylase réf. $\alpha$-Amylase A-1278 SIGMA.

(2) EDTA : Disodium éthylène diamine tétracétate crystal déshydraté "Reagent grade ".

(3) Sodium borate decahydrate "Reagent grade ".

(4) Sodium phosphate disodique p.a.

(5.1) Sodium lauryl sulfate.

(5.2) Ethoxy, ethanol (antimousse).

(6) Acide chlorhydrique normal p.a.

(7) Pepsine au 1/10 000 réf. 7197 Merck.

(8) Sodium acétate, $3 \mathrm{H}_{2} \mathrm{O}$ p.a.

(9) Acide acétique p.a.

(10) Cellulase, Celluclast $200 \mathrm{~L}$ type $\mathrm{N}$ de Novo Industrie (qui est un mélange de différentes enzymes de type cellulases et hémi-cellulases).

\section{c) Préparation des échantillons}

Après passage à l'étuve à $80^{\circ} \mathrm{C}$ maximum, les échantillons de maïs, hachés à l'ensileuse, sont broyés au broyeur à marteau une $1^{\text {re }}$ fois pour passer à travers une grille de $4 \mathrm{~mm}$ puis une $2^{\mathrm{e}}$ fois pour passer à travers une grille de $0,5 \mathrm{~mm}$.

\section{d) Mise en route de l'analyse}

Dans des sachets de nylon de dimension $6 \times 6 \mathrm{~cm}$, on verse $200 \mathrm{mg}$ de poudre préalablement séchée à l'étuve $24 \mathrm{~h}$ à $60^{\circ} \mathrm{C}$, on ajoute 2 billes de verre pour lester et par soudure on ferme le sachet.

Après un nouveau passage à l'étuve $24 \mathrm{~h}$ à $60^{\circ} \mathrm{C}$, on pèse le sachet plein soit $P_{1}$.

\section{e) Attaque à l'amylase}

\section{- Préparation du tampon}

- Dissoudre ensemble : $18,610 \mathrm{~g}$ de (2) et $6,81 \mathrm{~g}$ de (3) dans $500 \mathrm{ml}$ environ d'eau déminéralisée. 
- Dissoudre séparément 4,560 g de (4) dans $200 \mathrm{ml}$ environ d'eau déminéralisée.

- Mélanger les 2 solutions, ajouter $30 \mathrm{~g}$ de (5.1), compléter à $1 \mathrm{l}$.

- Ajouter $10 \mathrm{ml} \mathrm{de} \mathrm{(5.2).}$

Le $\mathrm{pH}$ de la solution doit être compris entre 6,9 et 7,1 .

\section{- Préparation de l'amylase}

- Préparer une solution d'amylase contenant $2 \mathrm{~g}$ pour $100 \mathrm{ml}$ d'eau déminéralisée.

- Ajouter $40 \mathrm{ml}$ de cette solution par 1 de tampon.

Mettre les sachets dans le bécher et verser la solution tampon + amylase dans la proportion de $20 \mathrm{ml}$ par sachet. Agiter. Mettre à l'étuve à $39{ }^{\circ} \mathrm{C}$ pendant $24 \mathrm{~h}$.

\section{f) Attaque à la pepsine}

- Préparer une solution de pepsine (7) à raison de $20 \mathrm{~g} / \mathrm{l}$ de $\mathrm{HCl}$ Normal (6).

- Sortir les sachets, rincer abondamment à l'eau tiède jusqu'à élimination de la mousse, faire un dernier rinçage à l'eau déminéralisée.

- Remettre les sachets dans le bécher.

- Verser la solution de pepsine dans le bécher dans la proportion de $20 \mathrm{ml}$ par sachet.

- Agiter. Mettre à l'étuve à $39^{\circ} \mathrm{C}$ pendant $24 \mathrm{~h}$.

\section{g) Attaque à la cellulase}

- Préparation du tampon.

* Dissoudre 7,90 g de sodium acétate (8) dans 11 d'eau contenant $2,46 \mathrm{ml}$ d'acide acétique (9).

* Le pH de ce tampon est de 4,8 $\pm 0,05$.

- Préparation de cellulase : ajouter $52,08 \mathrm{ml}$ de cellulase (10) par 1 de tampon.

- Sortir les sachets, laver abondamment à l'eau, rincer en dernier à l'eau déminéralisée.

- Remettre les sachets dans le bécher.

- Verser la solution de cellulase dans la proportion de $20 \mathrm{ml}$ par sachet.

- Agiter. Mettre à l'étuve à $50{ }^{\circ} \mathrm{C}$ pendant $48 \mathrm{~h}$. A.P.C.

h) Estimation de la digestibilité par la méthode

- Sortir les sachets, laver, rincer.

- Mettre les sachets à l'étuve à $60^{\circ} \mathrm{C}$ pendant $48 \mathrm{~h}$.

- Peser les sachets secs soit $P_{2}$.

- La quantité digérée est égale à $P_{1}-P_{2}$.

- Pourcentage de M.S. digestible $=\frac{P_{1}-P_{2}}{200} \times 100$.

Il est possible d'ajouter des "blancs" (sachets vides) dans la série ; la variation de poids de ces blancs à la suite des 3 attaques oscille entre 0 et $1 \mathrm{mg}$. On peut ou non en tenir compte pour effectuer une très légère correction.

On peut remarquer aussi qu'une attaque par la solution N.D.S. de Van Soest pendant $2 \mathrm{~h}$ à ébullition permet également de solubiliser l'amidon et les protéines. Cette méthode n'a pas été retenue en raison de difficultés d'identification des échantillons subissant ce traitement et surtout parce que, dans ces conditions, il y avait attaque des sachets de façon non uniforme.

\section{B. Expérimentation sur maïs}

Dans le cadre d'un essai réalisé en collaboration avec différents partenaires en 1983, 17 génotypes de maïs de bonne valeur agronomique, dont les témoins «Brulouis », "Bruex ", « Ery ", " Leader ", " Li i $:$ a » et «LG 11 », ce dernier sous forme normale et sous forme quasi isogénique $\mathrm{bm}_{3}$ (brown-midrib 3, GALLAIS et al., 1982) appelé pour des raisons de simplicité «LG $11 \mathrm{bm}_{3}$ », ont été étudiés en 6 lieux pour leur valeur nutritive.

Des essais en blocs, avec 3 répétitions de 2 lignes de $5 \mathrm{~m}$ ont été implantés sur 5 lieux dans la zone de culture des maïs précoces : Mons (Somme), Plelo (Côtesdu-Nord), Noyal (Ille-et-Vilaine), Illiers (Eure-et-Loir), Boigneville (Essonne). La densité de culture était comprise entre 93000 plantes/ha (Boigneville) et 106000 (Plelo). Des échantillons des parties " plante entière » et « tiges + feuilles » ont été prélevés et transmis après séchage à $80^{\circ} \mathrm{C}$ à Lusignan pour analyses.

A Lusignan, un essai en grande parcelle $\left(250 \mathrm{~m}^{2}, 2\right.$ répétitions, 85000 plantes/ha) a été réalisé, avec confection de micro-silos de maïs ensilage. La teneur en matière sèche moyenne à la récolte était de $33 \mathrm{p} .100$. Les ensilages ont été complémentés en urée dans la proportion de 15 p. 1000 en poids de la matière sèche. La proportion de grain dans l'ensilage a été estimée par un prélèvement de plantes au moment de la récolte. La digestibilité de ces ensilages a été estimée par les méthodes présentées ci-dessus et mesurée in vivo par des moutons maintenus en cages métaboliques (à raison de 4 à 6 moutons par silo). En raison d'un manque de disponibilité en semences, le génotype 5 n'a été étudié que sur un seul silo.

Au cours des traitements statistiques, les calculs ont été réalisés systématiquement avec et sans "LG 11 $b_{3}$ ", ce génotype étant hors type pour certains aspects qualité et conduisant donc à des estimations erronées des variances et des corrélations entre caractères. Les corrélations présentées sont les corrélations inter du modèle d'analyse de variance.

\section{RÉSULTATS ET DISCUSSION}

\section{A. Expérimentation sur maïs}

\section{Essai multilocal en petites parcelles}

Le tableau 1 présente les caractéristiques agronomiques des génotypes étudiés sur les différents lieux telles qu'elles nous ont été fournies par les expérimentateurs. Si les résultats de productivité et précocité «plante entière " paraissent corrects, ceux concernant le rendement de la partie "tiges + feuilles » paraissent plus douteux puisqu'ils conduisent à des rapports tiges + feuilles/plante entière très proches malgré des niveaux de maturité très différents $(0,45$ et 0,48 à 50 et 28 p. 100 de matière sèche).

L'analyse de variance des 4 caractères digestibilité A.P.C. " plante entière " et " tiges + feuilles ", et digestibilité jus de rumen "plante entière » et « tiges + feuilles » sur les différents lieux est donnée dans le tableau 2. Les effets génotypes et les effets lieux sont significatifs dans tous les cas. Les interactions géno- 
TABLEAU 1

Caractéristiques agronomiques des essais petites parcelles sur les différents lieux $(P E=$ plante entière $; T F=$ tige + feuilles $)$.

Agronomic data for small plot trials (yield and percent of dry-matter) $(P E=$ whole plant $; T F=$ stalk + leaves $)$.

\begin{tabular}{|c|c|c|c|c|}
\hline & \multicolumn{2}{|c|}{ Rendement t/ha } & \multirow{2}{*}{$\begin{array}{l}\text { Rapport } \\
\text { TF/PE }\end{array}$} & \multirow{2}{*}{$\begin{array}{l}0 \% \text { de } \\
\text { matière } \\
\text { sèche }\end{array}$} \\
\hline & $\mathrm{PE}$ & $\mathrm{TF}$ & & \\
\hline 1 Boigneville & 15,3 & 7,4 & 0,48 & 30,7 \\
\hline 2 Illiers & 11,9 & 5,4 & 0,45 & 50,4 \\
\hline 3 Noyal & 12,7 & 6,1 & 0,48 & 28,0 \\
\hline 4 Mons & 10,6 & 4,8 & 0,45 & 33,7 \\
\hline 5 Plelo & 11,1 & 3,7 & 0,33 & 31,7 \\
\hline
\end{tabular}

type lieux ne le sont que pour les mesures de digestibilité réalisées sur la partie " tiges + feuilles », et ceci avec ou sans " $\mathrm{LG} 11 \mathrm{bm}_{3}$ ».

Compte non tenu de " $\mathrm{LG} 11 \mathrm{bm}_{3}$ » qui présente une amélioration très notable de la valeur alimentaire, la variabilité mise en évidence pour les plantes entières est plus élevée avec la méthode jus de rumen $(\sigma=7,89)$ que celle mise en évidence avec la méthode A.P.C. $(\sigma=4,37)$. Cette différence ne se retrouve pas au niveau des tiges avec des écarts-types très proches (respectivement 7,00 et 6,61). La méthode A.P.C. paraît par ailleurs plus précise avec des écarts- types de l'erreur de 2,64 et 3,22 pour les parties " plante entière » et «tiges + feuilles», tandis que ceux obtenus avec la méthode jus de rumen valent respectivement 3,51 et 4,24 . Pour ces 2 méthodes, la précision des analyses faites sur "plante entière " paraît meilleure que celle des analyses faites sur la partie «tiges + feuilles ». Ceci rejoint d'autres observations réalisées au laboratoire et est sans doute dû au fait que la digestion de l'amidon et des protéines par l'amylase et la pepsine est bien réalisée, tandis que la dégradation des constituants pariétaux est plus imparfaitement réalisée. Ceux-ci ont en effet une digestibilité variable dans les différentes parties de la tige (donc risque d'erreur plus élevé par effet d'échantillonnage), car une lignification plus ou moins diffuse dans les tiges (GRAND, 1982) les préserve partiellement de la dégradation enzymatique. Enfin, l'erreur introduite par cette situation pour la partie «tiges + feuilles " se trouve diluée dans les analyses faites sur la plante entière où le grain représente de 30 à 50 p. 100 de la matière sèche.

Les interactions observées entre génotypes et lieux ne sont significatives que pour les analyses réalisées sur la partie "tiges + feuilles » et cela malgré une résiduelle plus élevée que pour les analyses réalisées sur la plante entière. Ceci est sans doute dû à des vitesses de lignification et/ou de dessèchement différentes de certains génotypes dans certains lieux, différences qu'estompe la présence d'amidon, dont la

TABLEAU 2

Essais multilocaux en petites parcelles - résultats des mesures de digestibilité et analyse de variance.

Multilocation small plot trials - results and analysis of variance.

\begin{tabular}{|c|c|c|c|c|c|c|c|c|}
\hline \multirow[b]{2}{*}{$\begin{array}{c}\text { Moyennes marginales } \\
\text { Génotypes }\end{array}$} & \multicolumn{4}{|c|}{ Plante entière } & \multicolumn{4}{|c|}{ Tige + feuilles } \\
\hline & \multicolumn{2}{|c|}{$\begin{array}{l}\text { Amylase }+ \text { pepsine } \\
+ \text { cellulase }\end{array}$} & \multicolumn{2}{|c|}{ Jus de rumen } & \multicolumn{2}{|c|}{$\begin{array}{c}\text { Amylase }+ \text { pepsine } \\
+ \text { cellulase }\end{array}$} & \multicolumn{2}{|c|}{ Jus de rumen } \\
\hline 1 Leader & \multicolumn{2}{|c|}{73,5} & \multicolumn{2}{|c|}{62,1} & \multicolumn{2}{|c|}{62,0} & \multicolumn{2}{|c|}{48,0} \\
\hline 2 & \multicolumn{2}{|c|}{71,2} & \multicolumn{2}{|c|}{59,9} & \multicolumn{2}{|c|}{61,9} & \multicolumn{2}{|c|}{47,0} \\
\hline 3 Ery & \multicolumn{2}{|c|}{72,2} & \multicolumn{2}{|c|}{62,6} & \multicolumn{2}{|c|}{63,7} & \multicolumn{2}{|c|}{51,5} \\
\hline 4 & \multicolumn{2}{|c|}{70,5} & \multicolumn{2}{|c|}{58,1} & \multicolumn{2}{|c|}{58,9} & \multicolumn{2}{|c|}{46,8} \\
\hline 5 & \multicolumn{2}{|c|}{73,3} & \multicolumn{2}{|c|}{62,9} & \multicolumn{2}{|c|}{64,1} & \multicolumn{2}{|c|}{50,4} \\
\hline 6 & \multicolumn{2}{|c|}{71,7} & \multicolumn{2}{|c|}{60,5} & & & & \\
\hline 7 Bruex & & & & & & & & \\
\hline 8 & & & & & & & & \\
\hline 9 & & & & & & & & \\
\hline 10 & & & & & & & & \\
\hline 11 & & & & & & & & \\
\hline 12 & & & & & & & & \\
\hline 13 Brulouis & & & & & & & & \\
\hline 14 Liza & & & & & & & & \\
\hline 15 & & & & & & & & \\
\hline 16 LG 11 & & & & & & & & \\
\hline $17 \mathrm{LG} 11 \mathrm{bm}_{3}$ & & & & & & & & \\
\hline Moyennes marginales & & & & & & & & \\
\hline lieux & $\mathrm{N}+\mathrm{bm}_{3}$ & $N$ & $\mathrm{~N}+\mathrm{bm}_{3}$ & $\mathrm{~N}$ & $\mathrm{~N}+\mathrm{bm}_{3}$ & $\mathrm{~N}$ & $\mathrm{~N}+\mathrm{bm}_{3}$ & $\mathrm{~N}$ \\
\hline 1 Boigneville & 71,2 & 70,6 & 58,3 & 57,9 & 62,8 & 62,0 & 48,6 & 47,9 \\
\hline 2 Illiers & 72,3 & 71,8 & 58,1 & 57,9 & 64,8 & 63,9 & 47,9 & 47,5 \\
\hline 3 Noyal & 70,8 & 70,6 & 60,5 & 60,2 & 58,5 & 57,9 & 46,3 & 45,6 \\
\hline 4 Mons & 73,2 & 72,9 & 66,6 & 66,1 & 66,1 & 65,5 & 53,3 & 53,2 \\
\hline 5 Plelo & 74,8 & 74,3 & 62,7 & 62,5 & 60,3 & 59,5 & 48,2 & 47,7 \\
\hline Analyse de variance & & & & & & & & \\
\hline F génotypes & $8,14^{* *}$ & $2,72 * *$ & $6,83^{* *}$ & $5,07^{* *}$ & $18,15^{* *}$ & $4,21^{* *}$ & $6,18^{* *}$ & $2,73^{* *}$ \\
\hline F lieux & $18,23^{* *}$ & $17,14^{* *}$ & $53,64^{* *}$ & $48,03 * *$ & $49,62 * *$ & $46,41 * *$ & $20,88^{* *}$ & $22,08^{* *}$ \\
\hline$F$ lieux $\times$ génotypes & 1,34 & 1,24 & 1,07 & 1,08 & $1,65^{* *}$ & $1,70^{* *}$ & $1,80^{* *}$ & $1,66^{* *}$ \\
\hline Ecart-type génotype & 7,62 & 4,37 & 9,05 & 7,89 & 13,69 & 6,61 & 10,31 & 7,00 \\
\hline Ecart-type erreur & 2,67 & 2,64 & 3,46 & 3,51 & 3,21 & 3,22 & 4,15 & 4,24 \\
\hline
\end{tabular}


digestibilité est très élevée et constante, dans la plante entière. On peut noter à ce propos que la digestibilité des plantes entières varie seulement de 70,6 à 74,3 p. 100 (méthode A.P.C.) et de 57,9 à 62,5 p. 100 (méthode jus de rumen) en moyenne de 16 génotypes sur chacun des 5 lieux, soit une variation de 3,7 et 4,6 points, alors que la teneur en matière sèche des plantes à la récolte varie de 28 à 50 p. 100 , ce qui correspond obligatoirement à des teneurs en amidon très variables. Dans ces mêmes conditions, la digestibilité de la partie tiges + feuilles varie de 7,6 points, soit environ le double de la variation observée pour la plante entière. La diminution de la qualité des tiges est, en tout ou partie, compensée par l'augmentation de la teneur en grain, mais l'augmentation de la teneur en grain ne conduit pas à une augmentation proportionnelle de la digestibilité, résultats qui confirment d'autres observations (DEMARQUILLY, 1969 ; GAllaIs et al., 1982).

La meilleure corrélation (tabl. 3) entre la méthode A.P.C. et la méthode jus de rumen est obtenue pour les analyses de la partie tiges + feuilles $\left(r=0,70^{* *}\right)$, celle observée pour les analyses sur plante entière est un peu plus faible $\left(\mathrm{r}=0,47^{* *}\right)$. Mais à ce stade, il est difficile d'affirmer qu'une des 2 méthodes est plus représentative de ce qui se passe in vivo, sur petits ou gros ruminants.

\section{Essais sur moutons}

Ces essais, présentés dans le tableau 4, ne permettent pas de mettre en évidence d'effets génotypes significatifs, sachant bien qu'il n'y a que 2 répétitions (2 silos par génotype, consommés chacun par 1 lot de moutons). L'écart-type génotypique mis en évidence par les animaux est très faible pour le C.U.D. (coefficient d'utilisation digestive) de la matière organique,
TABLEAU 3

Corrélations entre les mesures de digestibilité réalisées sur plante entière (PE) et sur la partie tiges + feuilles (TF) avec les méthodes $A P C$ et jus de rumen $\left(N+b m_{3}=\right.$ avec $L G 11 b m_{3} ; N=$ sans $L G\left(/ b_{3}\right)$.

Correlations between results obtained on whole plant (PE) and stalks + leaves (TF) with $A P C$ and rumen juice methods $\left(N+b m_{3}=\right.$ with $L G M b m_{3} ; N=$ without $\left.L G 11 b m_{3}\right)$

\begin{tabular}{|c|c|c|c|c|c|c|c|}
\hline & & $\mathrm{APC}$ & PE & J. rumen & PE & APC & $\mathrm{TF}$ \\
\hline & & $\mathrm{N}+\mathrm{bm}_{3}$ & $\mathrm{~N}$ & $\mathrm{~N}+\mathrm{bm}_{3}$ & N & $\mathrm{N}+\mathrm{bm}_{3}$ & $\mathrm{~N}$ \\
\hline $1 \mathrm{APC}$ & PE & - & - & & & & \\
\hline $2 \mathrm{~J}$. rumen & $\mathrm{PE}$ & $0,49^{* *}$ & $0,47^{* *}$ & - & - & & \\
\hline $3 \mathrm{APC}$ & $\mathrm{TF}$ & $0,42 * *$ & 0,11 & $0,33^{* *}$ & 0,22 & - & - \\
\hline $4 \mathrm{~J}$. rumen & $\mathrm{TF}$ & $0,34^{* *}$ & 0,14 & $0,43^{* *}$ & $0,38^{* *}$ & $0,74 * *$ & $0,70^{* *}$ \\
\hline
\end{tabular}

alors qu'il est 3,6 fois plus élevé pour le C.U.D. de la cellulose brute. Il y a de plus une corrélation négative significative $\left(r=-0,53^{*}\right)$ (tabl. 5) entre le C.U.D. de la cellulose brute et la teneur de l'ensilage en grain. Ces résultats confirment qu'une augmentation de la teneur en grain conduit à une moins bonne digestibilité de la cellulose au niveau du rumen, parce que la flore est modifiée (EL SHAZY et al., 1961) et parce que les tiges des maïs sont de plus mauvaise qualité après des transferts plus importants vers l'épi.

Les corrélations entre la digestibilité mesurée par les moutons et les digestibilités mesurées par les méthodes jus de rumen ou A.P.C. ne sont pas élevées (tabl. 5), entre 0,11 et 0,45 (sans « LG 11 bm $_{3} »$ ), avec une tendance à une meilleure corrélation pour la méthode jus de rumen. Ces corrélations, non significatives, sont toutefois discutables puisque les effets génotypes mesurés par les moutons sont également non significatifs.

TABLEAU 4

Essais grandes parcelles et mesures de digestibilité sur moutons - résultats et analyse de variance.

Large plot trials, and in vivo sheep measures of digestibility - results and analysis of variance.

\begin{tabular}{|c|c|c|c|c|c|c|c|c|c|c|}
\hline & \multicolumn{2}{|c|}{ CUD M. Organique } & \multicolumn{2}{|c|}{ CUD Cellulose brute } & \multicolumn{2}{|c|}{ Digestibilité APC } & \multicolumn{2}{|c|}{$\begin{array}{l}\text { Digestibilité } \\
\text { jus de rumen }\end{array}$} & \multicolumn{2}{|c|}{ Teneur en grain } \\
\hline 1 Leader & \multicolumn{2}{|c|}{73,3} & \multicolumn{2}{|c|}{50,0} & \multicolumn{2}{|c|}{84,2} & \multicolumn{2}{|c|}{69,4} & \multicolumn{2}{|c|}{48,8} \\
\hline 2 & \multicolumn{2}{|c|}{71,2} & \multicolumn{2}{|c|}{48,5} & \multicolumn{2}{|c|}{79,7} & \multicolumn{2}{|c|}{63,6} & \multicolumn{2}{|c|}{51,1} \\
\hline 3 Ery & \multicolumn{2}{|c|}{70,3} & \multicolumn{2}{|c|}{46,7} & \multicolumn{2}{|c|}{82,0} & \multicolumn{2}{|c|}{66,4} & \multicolumn{2}{|c|}{53,4} \\
\hline 4 & \multicolumn{2}{|c|}{70,7} & \multicolumn{2}{|c|}{49,2} & \multicolumn{2}{|c|}{76,9} & \multicolumn{2}{|c|}{66,9} & \multicolumn{2}{|c|}{48,1} \\
\hline 5 & \multicolumn{2}{|c|}{73,0} & \multicolumn{2}{|c|}{56,7} & \multicolumn{2}{|c|}{81,0} & \multirow{2}{*}{\multicolumn{2}{|c|}{69,9}} & \multicolumn{2}{|c|}{51,0} \\
\hline 6 & \multicolumn{2}{|c|}{71,1} & \multicolumn{2}{|c|}{55,2} & & & & & & \\
\hline 7 Bruex & & & & & & & & & & \\
\hline 8 & & & & & & & & & & \\
\hline 9 & & & & & & & & & & \\
\hline 10 & & & & & & & & & & \\
\hline 11 & & & & & & & & & & \\
\hline 12 & & & & & & & & & & \\
\hline 13 Brulouis & & & & & & & & & & \\
\hline 14 Liza & & & & & & & & & & \\
\hline 15 & & & & & & & & & & \\
\hline 16 LG 11 & & & & & & & & & & \\
\hline $17 \mathrm{LG} 11 \mathrm{bm}_{3}$ & & & & & & & & & & \\
\hline Analyse de variance & & & & & & & & & & \\
\hline & $\mathrm{N}+\mathrm{bm}_{3}$ & $\mathrm{~N}$ & $\mathrm{~N}+\mathrm{bm}_{3}$ & $N$ & $\mathrm{~N}+\mathrm{bm}_{3}$ & $\mathrm{~N}$ & $\mathrm{~N}+\mathrm{bm}_{3}$ & $\mathrm{~N}$ & $\mathrm{~N}+\mathrm{bm}_{3}$ & $\mathrm{~N}$ \\
\hline F génotypes & 1,69 & 1,26 & 2,45 & 1,42 & 1,80 & 1,00 & 2,02 & 1,29 & 2,09 & 2,12 \\
\hline Ecart-type génotype & 1,62 & 1,45 & 6,74 & 5,25 & 3,56 & 2,75 & 3,33 & 2,76 & 3,71 & 3,83 \\
\hline Ecart-type erreur & 1,25 & 1,30 & 1,36 & 1,39 & 2,66 & 2,76 & 2,34 & 2,43 & 2,57 & 2,64 \\
\hline
\end{tabular}


TABLEAU 5

Corrélations entre les mesures de digestibilité obtenues in vivo (moutons) et in vitro (APC et jus de rumen).

Correlation between in vivo (sheep) and in vitro (APC, rumen juice) tests of digestibility.

\begin{tabular}{|c|c|c|c|c|c|c|c|c|}
\hline & \multicolumn{2}{|c|}{ CUD MO } & \multicolumn{2}{|c|}{ CUD Cellulose } & \multicolumn{2}{|c|}{ A P C } & \multicolumn{2}{|c|}{ J. rumen } \\
\hline & $\mathrm{N}+\mathrm{bm}_{3}$ & $\mathrm{~N}$ & $\mathrm{~N}+\mathrm{bm}_{3}$ & $\mathrm{~N}$ & $\mathrm{~N}+\mathrm{bm}_{3}$ & $\mathrm{~N}$ & $\mathrm{~N}+\mathrm{bm}_{3}$ & $N$ \\
\hline \multicolumn{9}{|l|}{ CUD M. Organique } \\
\hline CUD Cellulose & 0,44 & 0,20 & - & - & & & & \\
\hline Digestibilité APC & $0,48^{* *}$ & 0,25 & $0,49^{*}$ & 0,11 & - & - & & \\
\hline Digestibilité J. rumen & $0,60^{* *}$ & 0,45 & $0,58 * *$ & 0,32 & $0,58 * *$ & 0,30 & - & - \\
\hline Teneur en grain & 0,03 & 0,05 & $-0,43$ & $-0,53^{*}$ & 0,15 & 0,23 & 0,06 & 0,10 \\
\hline
\end{tabular}

\section{B. Essais sur sorgho et fétuque élevée}

Avec des sorghos de $1^{\text {er }}$ cycle, les corrélations mesurées sur 8 génotypes entre le C.U.D. de la matière sèche mesurée sur mouton et, respectivement, les digestibilités mesurées par les méthodes A.P.C. et jus de rumen sont de 0,83 et 0,76 , la corrélation entre les digestibilités A.P.C. et jus de rumen étant de 0,84 . Sur des sorghos de $2^{\text {e }}$ cycle, où la variabilité pour la qualité entre génotypes s'exprime peu, ces 3 corrélations sont respectivement de $0,65,0,38$ et 0,84 .

Avec des fétuques, pour 5 génotypes et 4 années d'observations, les corrélations entre le C.U.D. de la matière sèche mesuré sur moutons et, respectivement, les digestibilités mesurées par les méthodes A.P.C. et jus de rumen sont en moyenne de $0,85(0,74$ à 0,32$)$ et $0,73(0,60$ à 0,84$)$, la corrélation entre les digestibilités A.P.C. et jus de rumen valant en moyenne $0,77(0,52$ à 0,89 ). Sur des fétuques de $2^{\mathrm{e}}$ cycle, où là encore la variabilité pour la qualité entre génotypes s'exprime peu, l'écart mini-maxi étant dans ce cas divisé par 3, ces 3 corrélations sont très variables selon les années puisqu'elles sont comprises respectivement entre 0,03 à $0,93,0,01$ à 0,88 et 0,07 à 0,95 .

La précision des mesures observées sur les échantillons de fétuque est meilleure pour la méthode A.P.C. que pour la méthode jus de rumen. Ainsi, pour un groupe d'échantillons donné et lors de plusieurs séries de mesures, l'écart-type entre séries est de $\pm 0,06$ pour la méthode A.P.C. et $\pm 1,9$ pour la méthode jus de rumen. De même, pour un échantillon donné, répété dans une série de mesures, l'écart-type de la digestibilité est de $\pm 0,5$ pour la méthode A.P.C. et de 1,4 pour la méthode jus de rumen.

\section{CONCLUSIONS}

\section{A. Au niveau du laboratoire d'analyse}

Sur le plan de la manipulation, la méthode jus de rumen présente de nombreux inconvénients. Elle impose l'entretien d'animaux fistulés maintenus en bonne santé, avec une alimentation très équilibrée. Des conditions très strictes de température, d'absence d'oxygène au moment du prélèvement et de la mise en route de l'analyse doivent être observées. En revanche, le coût unitaire d'une détermination est peu élevé, compte non tenu des frais d'entretien des animaux.
La méthode A.P.C. en dehors du coût relativement élevé des enzymes, présente 2 avantages principaux. Les différents tampons sont de fabrication aisée et les différentes étapes de l'analyse sont réalisées sans filtration.

Sur le plan des résultats, la méthode A.P.C. est plus précise avec des écarts-types sur une mesure toujours inférieure à 1, tandis qu'ils sont d'environ 1,8 pour la méthode jus de rumen. Une mauvaise fidélité caractérise la méthode jus de rumen, avec des corrélations entre 2 séries de mesure variant de 0,9 à 0,7 alors qu'elles ne sont jamais inférieures à 0,9 pour la méthode A.P.C. De plus, il est possible d'obtenir de meilleurs étalonnages sur spectrophotomètre dans le proche infrarouge pour les valeurs de digestibilité obtenues par la méthode A.P.C. que pour celles obtenues par la méthode jus de rumen.

\section{B. Au niveau du laboratoire de sélection}

L'utilisation des "moutons castrés standards " n'est pas envisageable sur un grand nombre d'échantillons, même s'ils sont considérés comme outil de référence zootechnique. De plus, si ces animaux, qui ne sont pas en production, permettent effectivement de mesurer une digestibilité de la matière organique in vivo, les performances réalisées par des taurillons en croissance ou des vaches laitières en production ne résultent pas seulement de la digestibilité des fourrages qui leur sont offerts, mais aussi des quantités ingérées et de l'importance de la qualité des compléments azotés et énergétiques qui sont apportés. Ils permettent effectivement de mettre en évidence des différences intraspécifiques ou intervariétales élevées, discriminent bien des types un peu extrêmes comme maïs normal, maïs « $\mathrm{bm}_{3} »$ (GALlaIS et al., 1982) ou fétuque classique, fétuque souple (JADAS-HECART, 1982 ; GILLET et al., 1983) mais sont peu aptes à mettre en évidence des différences faibles (maïs normaux génétiquement proches ou graminées de $2^{\mathrm{e}}$ coupe), en particulier dans les cas d'essais à peu de répétitions.

La méthode A.P.C. permet de trier un grand nombre de génotypes et cela d'autant plus qu'elle permet de meilleurs étalonnages pour la technique du proche infrarouge. Ses résultats sont tout à fait satisfaisants pour des fourrages classiques, pauvres en amidon, et sont au moins aussi bien corrélés avec les mesures faites sur animaux que la méthode jus de rumen. Pour des fourrages très riches en amidon comme le maïs, la méthode A.P.C. permet d'estimer une digestibilité 
potentielle de la matière organique, en digérant en particulier tous les constituants pariétaux digestibles quelle que soit la teneur en amidon; ce qui n'est réalisé ni par la méthode jus de rumen, ni par les animaux puisque la cellulose n'est effectivement bien digérée par le ruminant que lorsque l'amidon n'est pas trop abondant. Ceci explique d'ailleurs que les corrélations digestibilité A.P.C. avec digestibilité jus de rumen ou digestibilité in vivo puissent être faibles dans certains cas. L'objectif de la sélection maïs fourrage est de trier des génotypes dont la digestibilité de la plante entière soit la plus élevée possible indépendamment de la teneur en amidon. Cet objectif ne peut être atteint ni par les études sur moutons, qui ne sont pas envisageables sur des grands effectifs, ni par la méthode jus de rumen qui fournit une estimation proche de ce qui est obtenu avec des ruminants, mais il est approché par la méthode A.P.C. L'hypothèse est alors faite que des génotypes ainsi triés seront effectivement utilisés de façon optimale par des ruminants, même s'ils sont dans certains cas sous-utilisés, les expérimentations faites avec le matériel « $b m_{3}$ » montrant clairement que les ruminants peuvent répondre par une augmentation des quantités ingérées quand la digestibilité des constituants pariétaux qui leur sont offerts est améliorée (GALLAIS et al., 1982; BARRIĖRE et al., 1985 ; HODEN et al., 1985 ; MALTERRE et al., 1985).
Un complément utile à la méthode A.P.C. serait de disposer d'un test de routine, étalonnable dans le proche infra-rouge, des teneurs en amidon. En effet, actuellement, à digestibilité égale, il n'est pas possible de faire la différence entre un génotype à teneur en amidon plus élevée et un génotype dont les constituants pariétaux sont plus digestibles, et cela quelle que soit la méthode de digestibilité employée. Or, c'est bien en améliorant la digestibilité apparente des constituants pariétaux que les progrès les plus notables en digestibilité du maïs fourrage sont attendus, amélioration qui peut être due à des modifications chimiques de la cellulose, à la modification du rapport hémi-cellulose/cellulose, à une réduction de la teneur en lignine ou à une modification de sa structure qui rendrait la cellulose plus disponible.

Ce qui importe finalement pour la sélection, c'est d'avoir une méthode en routine qui mette en évidence des progrès nets, qui permette d'éliminer les génotypes de mauvaise qualité et de vérifier qu'il n'y a pas une perte corrélative de qualité au cours de cycles d'amélioration $\mathrm{du}$ rendement. La méthode A.P.C. nous semble être un outil intéressant pour répondre à ces objectifs, aussi bien sur les fourrages classiques que sur les fourrages plus riches en amidon.

Reçu le 17 décembre 1984. Accepté le 14 novembre 1985.

\section{RÉFÉRENCES BIBLIOGRAPHIQUES}

Aufrère J., 1982. Etude de la prévision de la digestibilité des fourrages par une méthode enzymatique. Ann. Zootech., 31 (2), 111-130.

Demarquilly $C_{\text {., }}$ 1969. Valeur alimentaire du maïs fourrage. I. Composition chimique et digestibilité du maiss sur pied. Ann. Zootech., 18, 17-32.

Barrière Y., Gallais A., Huguet L., Traineau R., 1985. Le maïs brown-midrib plante entière. (1) Caractéristiques générales. Bull. Tech. C.R.Z.V., Theix, I.N.R.A., $\mathrm{n}^{\circ}$ 60, 43-44.

El Shazy K., Dehority B. A., Johnson R. R., 1961. Effect of starch on the digestion of cellulose in vitro and in vivo by rumen microorganisms. J. Anim. Sci., 20, 268-273.

Gallais A., Vincourt P., Huguet L., 1982. Objectifs et critères de sélection du maîs fourrage. Congrès Eucarpia, Montreux, Suisse, septembre 1981, $35 \mathrm{p}$.

Gillet M., Noël C., Jadas-Hécart J., 1983. La cafétéria d'auges, méthode d'étude de l'appétibilité des fourrages. Agronomie, 3 (8), 817-822.

Grand C., 1982. Natural variations and controlled changes in lignification process. Holzforschung, 36, 217-223.
Hoden A., Huguet L., Journet M., Barrière Y., Gallais A., Mourguet A., 1985. Le maïs brown midrib plante entière. Utilisation sous forme d'ensilage par les vaches laitières. Bull. Tech. C.R.Z.V., I.N.R.A., Theix, $\mathrm{n}^{\circ} 60,51-58$.

Jadas-Hécart J., 1982. Etude en pâture de l'appétibilité de la fétuque élevée (Festuca arundinacea Schreb.) à l'aide de moutons. Agronomie, 2 (5), 487-492.

Lila M., 1977. Technique de mesure de la digestibilité "in vitro" en grande série en vue de la sélection pour la qualité des plantes fourragères. Ann. Amélior. Plantes, 27 (1), 117-128.

Mac Dougall E. I., 1948. Studies on ruminant saliva. I. Composition and output of sheep's saliva. Biochem. J., 43, 99-109.

Malterre C., Bertin G., Gallais A., Huguet L., Micol D., 1985. Le maïs brown-midrib plante entière 2 . Utilisation sous forme d'ensilage par les taurillons. Bull. Tech. C.R.Z.V., I.N.R.A., Theix, $n^{\circ} 60,44-51$.

Tilley J. M. A., Terry R. A., 1963. A two stage technique for the " in vitro " digestion of forage crops. J. Br. Grassl. Soc., 18, 104-111. 